\title{
Neoadjuvant and Adjuvant Therapy in Operable Pancreatic Cancer: Both Honey and Milk (but No Bread?)
}

Kjetil Søreide (D)

Received: November 24, 2020 / Accepted: December 18, 2020 / Published online: January 13, 2021

(C) The Author(s) 2021

\begin{abstract}
Pancreatic cancer has a dismal prognosis. Resection is the best option for cure, supported by multimodal therapy to treat the systemic disease. While adjuvant therapy has become standard in those who are fit and who can tolerate the given regimen, the concept of perioperative (neoadjuvant) therapy is building momentum. The concepts of "borderline" and "locally advanced" have changed the previous dichotomized "resectable/non-resectable" into subcategories for which new algorithms have emerged, with neoadjuvant therapy discussed both for upfront resectable pancreatic cancer, for those deemed borderline resectable, and as "induction or conversion" therapy for locally advanced disease. The purpose of this invited commentary is to discuss some of the changing paradigms in multimodal therapy for operable pancreatic cancer. The PREOPANC trial presented randomized data on the role of
\end{abstract}

K. Søreide $(\square)$

-Department of Gastrointestinal Surgery, Stavanger

University Hospital, Stavanger, Norway

e-mail: ksoreide@mac.com

K. Søreide

Department of Clinical Medicine, University of

Bergen, Bergen, Norway

K. Søreide

Gastrointestinal Translation Research Unit,

Stavanger University Hospital, Stavanger, Norway neoadjuvant therapy for resectable and borderline cancers, but new questions have emerged. The role of combination therapy in the preoperative setting is discussed in the light of this trial. FOLFIRINOX has emerged as the most potent treatment regimen in the metastatic and adjuvant setting, but with no level I data to support neoadjuvant use yet. Several trials are ongoing to arrive at the best answer.

Keywords: Chemotherapy; Folfirinox; Gemcitabine; Neoadjuvant randomized trial; Outcome; Pancreatic cancer; Resection rate; Surgery; Survival

Pooh always liked a little something and he was very glad to see Rabbit getting out the plates and mugs; and when Rabbit said: "Honey or condensed milk with your bread?" he was so excited that he said, "Both," and then, so as not to seem greedy, he added, "but don't bother about the bread, please." from Winnie-the-Pooh by A. A. Milne.

\section{DIGITAL FEATURES}

This article is published with digital features to facilitate understanding of the article. To view digital features for this article go to https://doi. org/10.6084/m9.figshare.13536473. 


\section{INTRODUCTION}

Pancreatic cancer is projected to become a leading cause of cancer-related morbidity and death worldwide $[1,2]$. With very limited progress overall, and scarcely noticeable effects on long-term survival over the past 50 years [3], it is only in the last decade that some remarkable changes in survival times have been noted with novel multimodal management introduced into the multimodal care pathway. While surgery is the single most important factor for achieving long-term survival, this is hardly achieved without systemic therapy. Also, surgery is offered to less than $20 \%$ of all patients with pancreatic cancer. So pancreatic surgeons may become a little eager, just like Winnie-thePooh (quote above), for "a little something" and to have the "honey and milk" too, when there appears to be a resectable cancer. Should one have chemo-(radio)therapy too, and also, even after the surgery?

Evolving with evidence along several randomized trials, adjuvant therapy after resection has become the standard [4], demonstrated among others through the series of European Study Group for Pancreatic Cancer (ESPAC) trials [5-8]. Clearly, introduction of novel agents, including gemcitabine, nab-paclitaxel and combined regimens such as FOLFIRINOX (i.e., folinic acid, fluorouracil, irinotecan and oxaliplatin), have provided more effective therapy, first demonstrated in metastatic pancreatic cancer [9] and later as adjuvant therapy in the Unicancer and Canadian Cancer Trials Group (CCTG) PRODIGE 24/CCTG PA6 trial, with a median overall survival reported at an unprecedented 54.4 months after surgery [10].

Moreover, the concepts of "borderline" and "locally advanced" have changed the previous dichotomized "resectable/non-resectable" into subcategories for which new algorithms have emerged, with neoadjuvant therapy discussed both for upfront resectable disease, for those deemed borderline resectable, and as "induction or conversion" therapy for locally advanced disease (Fig. 1), with subsequent exploration and resection in responders [11-13]. However, there is considerable debate over the role and need for upfront chemotherapy in otherwise resectable pancreatic cancer. This is partly related to the knowledge that resection is an essential component for chance of cure, and also increasing experience with extended resections, including safer resections in the case of portal vein involvement $[14,15]$. However, reports on early recurrences despite "successful" radical surgery suggest pancreatic cancer to be a highly systemic disease even from the early onset.

Thus, when new and more effective therapy becomes available, it is tempting to want it all, like Winnie-the-Pooh's craving for both milk and honey. Certainly, the milk and honey analogy can be played out to contemporary treatment strategies for clinically resectable or borderline-resectable pancreatic cancer (Fig. 2). However, which treatment strategy to choose over the other may not be immediately evident from the lack of available level 1 data [16].

Also, a plethora of new definitions and strategies have emerged [12]. Indeed, use of neoadjuvant therapy has crept into many institutional protocols despite lack of data. Recent meta-analyses suggest a benefit from neoadjuvant therapy, but with low-quality data based on institutional series, lack of a control arm and failure to account for dropouts of therapy for an intention-to-treat analysis [17] (Fig. 3). Yet, still, opinions and practice are largely split between "surgery first" and "neoadjuvant therapy" attitudes, due to the lack of good level 1 data to clearly support one opinion over the other. Proponents for surgery argue that " $a$ chance to cut is a chance to cure," or at least to achieve long-term survival for some patients, and progression under neoadjuvant therapy would deprive patients of this chance for surgical cure. In contrast to this view, those in favor of a neoadjuvant therapy approach first use the argument that patients with early recurrence (e.g., $<6$ months after surgery) are those who are unlikely to have had any benefits from the surgery, other than the likely added morbidity and possibly complications from the procedure. Also, early recurrence is associated with a very poor prognosis (Fig. 4) [18]. Even if the time to the start of adjuvant therapy is delayed by postoperative complications, the 


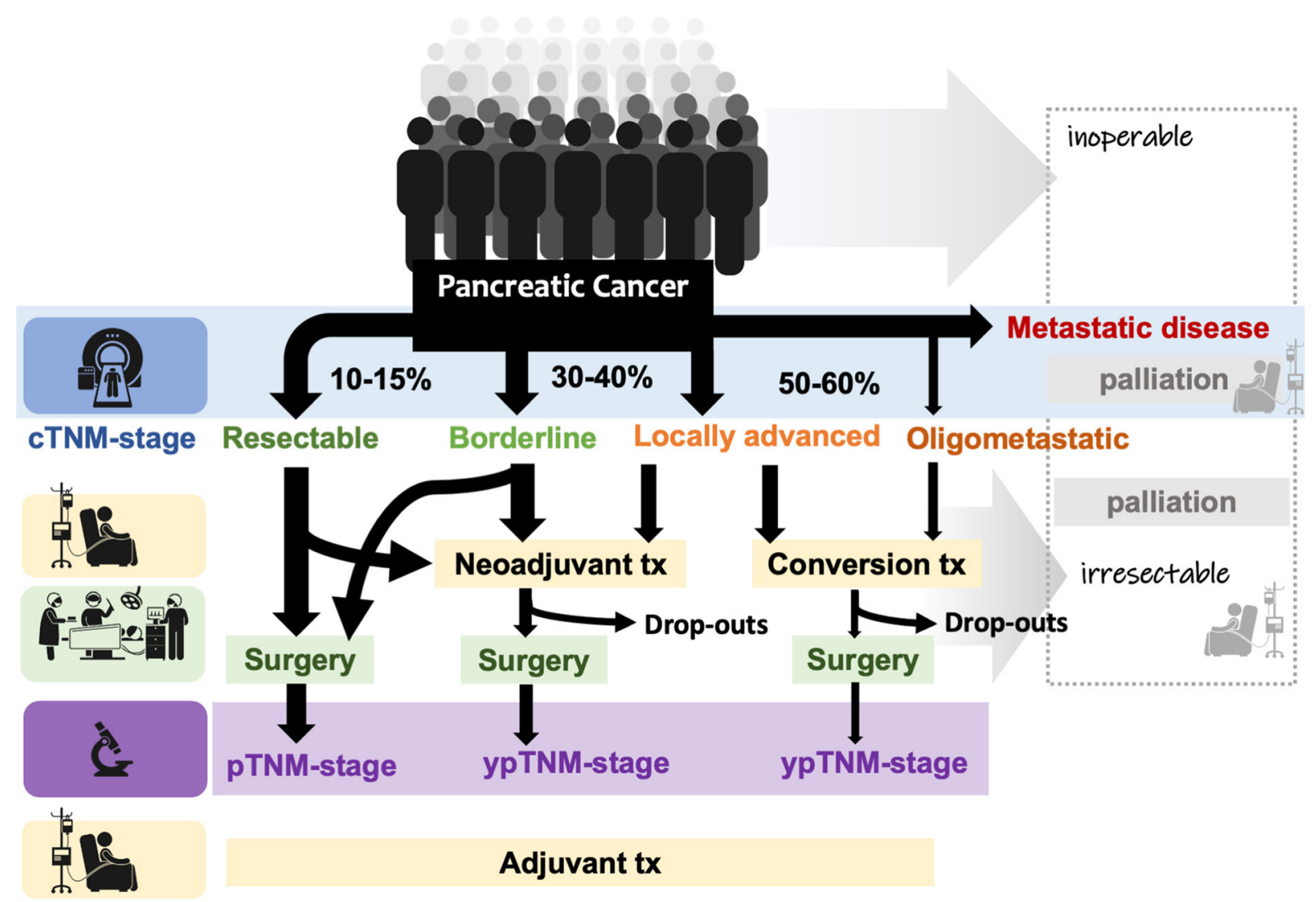

Fig. 1 The contemporary patient and treatment landscape in pancreatic cancer. Reproduced and slightly modified with permission under the Creative Commons CC-BY-

NC-ND from Eur J Surg Oncol. 2020;46(8):1554-7 (C)2020 The Authors

most important factor is the ability to complete the course of adjuvant chemotherapy [19]. Notably, less than $60 \%$ of patients receive adjuvant chemotherapy after surgery-for numerous reasons-and hence do not receive multimodal oncological care $[20,21]$. Thus, giving the therapy before surgery would just seem reasonable, as it would hopefully increase the numbers who receive systemic therapy.

\section{The Dutch PREOPANC Trial}

With this as a backdrop, the Dutch PREOPANC trial was designed several years ago, with ethics approval in 2012 [22]. The trial commenced in 2013 and successfully randomized 248 patients by July 2017, involving 16 Dutch hospitals. The trial was powered to show a 6-month survival benefit with preoperative chemoradiotherapy

over upfront surgery (expected 17 months to 11 months, respectively) in the planned intention-to-treat analysis. Somewhat disappointingly, and maybe even to some a bit surprisingly, the study reported no significant difference between the two arms [23]: median overall survival by intention to treat was 16.0 months with preoperative chemoradiotherapy and 14.3 months with immediate surgery $(P=0.096)$.

That being said, and with the trial design and endpoint to power the sample size in mind, one should not disregard the favorable outcomes for several of the secondary endpoints and subgroups. For example, the R0 rate was better with neoadjuvant therapy. Also, the neoadjuvant arm noted better disease-free survival and locoregional failure-free interval, as well as significantly lower rates of pathologic lymph 

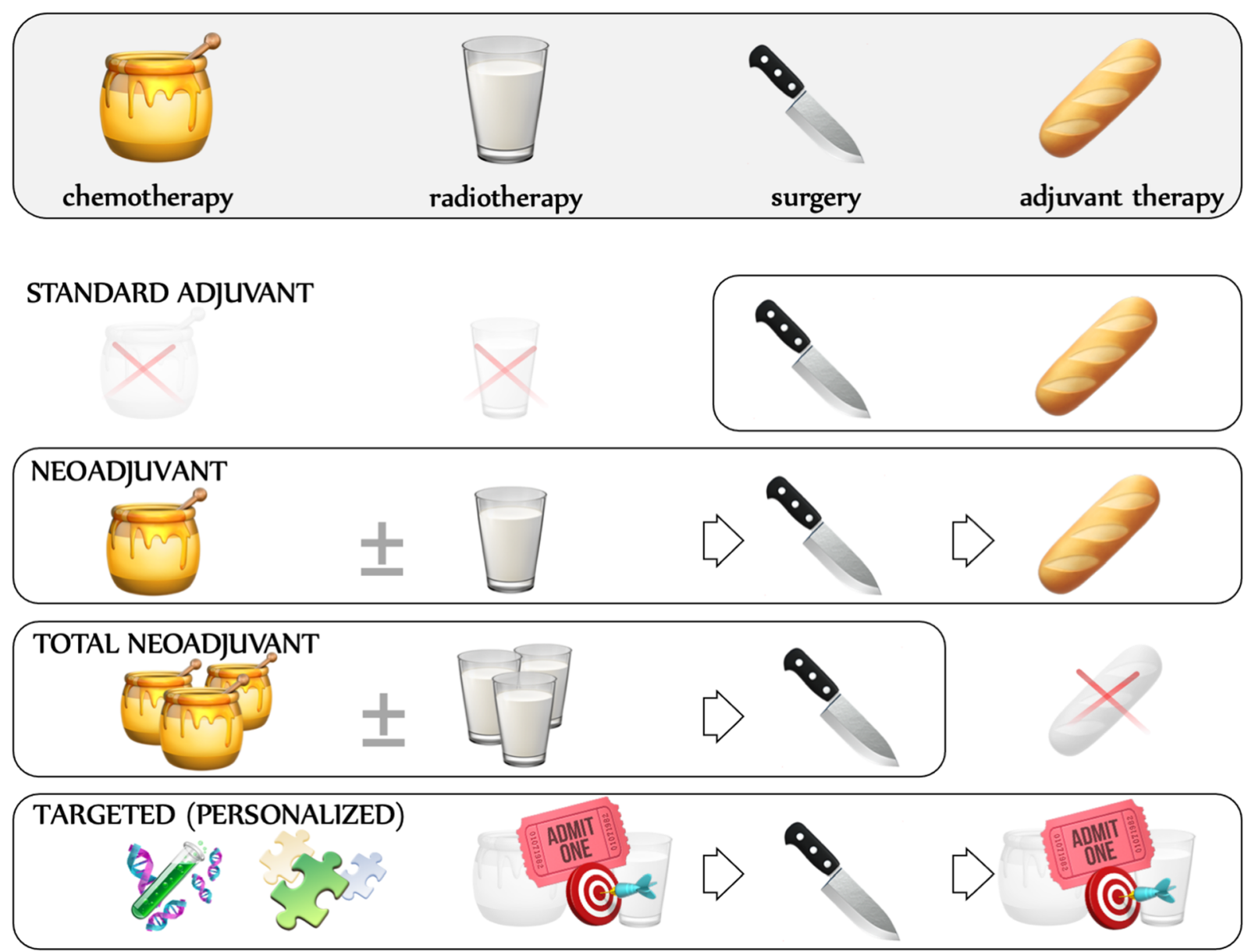

Fig. 2 Analogy to the different treatment strategies in (borderline) resectable pancreatic cancer. A "honey, milk or bread" analogy, from Winnie-the-Pooh. All or select few?

nodes, perineural invasion and venous invasion. However, all these endpoints need to be confirmed both for return on overall survival and for their applicability for use as surrogate markers for improved oncological control in appropriately designed trials.

\section{Pearls and Pitfalls to Consider}

A few things should be kept in mind when reviewing the PREOPANC trial data (Fig. 5). The trial was designed to investigate whether preoperative radiochemotherapy improves overall survival by means of increased radical (R0) resection rates in patients with resectable or borderline-resectable
At the bottom, the future personalized approach is pictured based on a complete workup of specific factors and targeted therapies tailored to the individual

Obviously, one should keep in mind the time and the setting during which the trial was designed (now almost a decade in the passing). It is easy to forward critique in hindsight-it is much harder to design, launch and execute a trial like the PREOPANC trial, requiring the buyin from several disciplines and across multiple institutions. For this effort the investigators should be highly commended and applauded. Designing trials will always be a challenge, as emerging data and new experience during the trial period may change the knowledge landscape within which a trial was designed and for which it was intended to provide new answers. Hence, the Dutch Pancreatic Cancer Group has led the way in this national, multicenter trial by 


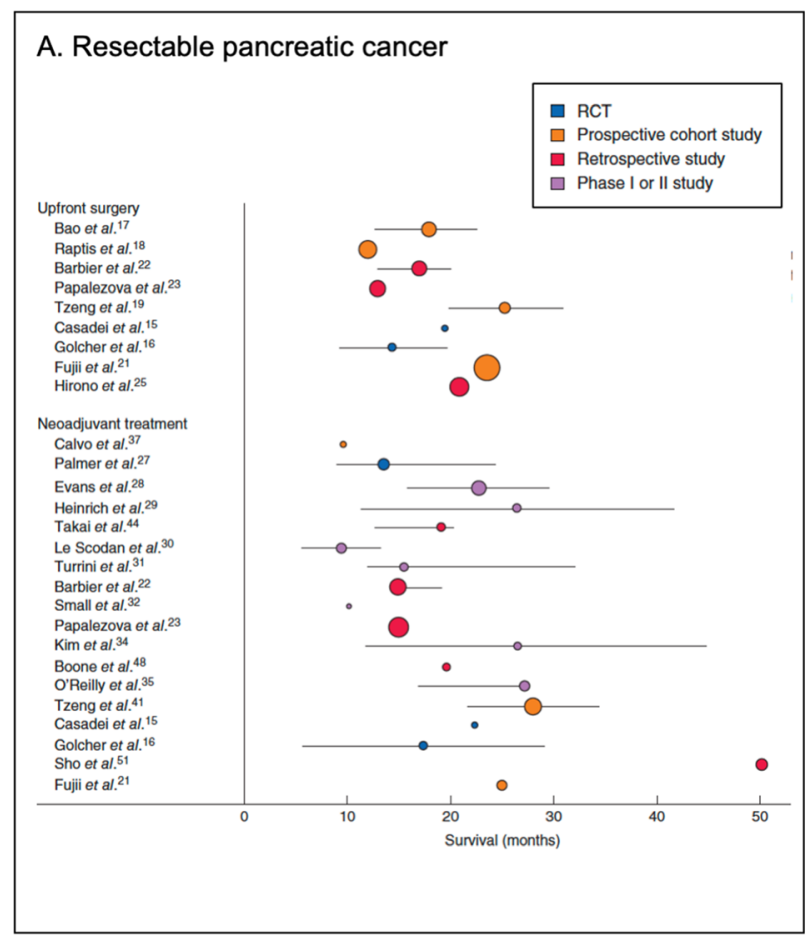

Fig. 3 Comparing overall survival between studies. Reported median overall survival with 95\% confidence intervals. a Resectable pancreatic cancer. (b) Borderline pancreatic cancer. Reproduced with permission under the

showing how a trial has involved several institutions within a country to participate in a surgical oncology trial.

Nonetheless, some remarks may be discussed as a backdrop to the results from the PREOPANC study. First, the eligibility and inclusion criteria may be considered. While a Karnofsky performance status $\geq 70$ (or WHO performance status of 0 or 1 ) is not controversial-patients must be able to tolerate the treatment giventhe inclusion of cancers located both in the head and in the body/tail of the gland may to some degree contribute to the results achieved in the trial. Notably, several studies suggest that body and tail cancers are biologically different from those located in the pancreatic head. This has been demonstrated at the genetic level, through immune profiling and regarding clinical outcomes [24-27]. Although distal cancers made up a minority (8\% in the surgery first compared to $18 \%$ in the neoadjuvant group) [23], it may potentially have influenced the

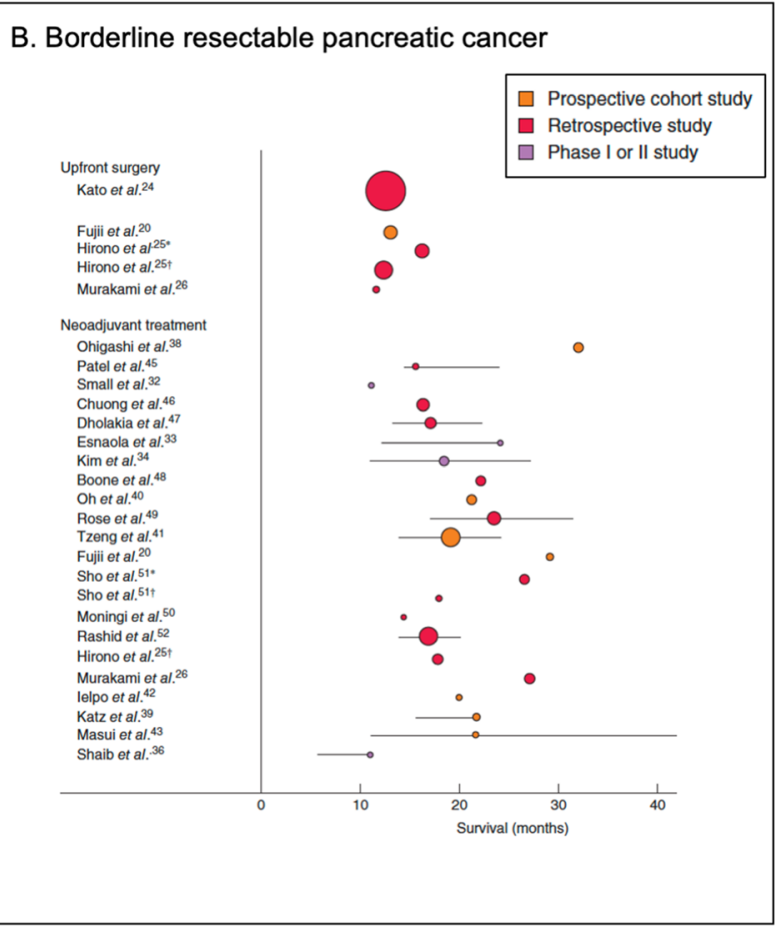

Creative Commons CC-BY-NC-ND from Versteijne et al. [17] Br J Surg. 2018;105(8):946-58 Wiley (C2018 The Authors

results. However, location of tumor was noted to have no significant difference in median overall survival times in a Dutch populationbased cohort [28]. Further, with regard to the PREOPANC outcomes, one should note that cT1 tumors $(<2 \mathrm{~cm}$ on clinical imaging/staging) were not included in the trial $[22,23]$. In general, the prognosis is better with these "earlystage" T1 cancers compared to higher stages, but at the same time even such early pancreatic cancers have an overall very high risk of metastasis-much higher than any other earlystage solid cancer of similar size-demonstrating a high likelihood for systemic disease even in the earliest stages of pancreatic cancer $[29,30]$. Thus, the notion that pancreatic cancers show a systemic disease behavior from an early onset and with the smallest of tumor sizes (even $<0.5 \mathrm{~cm}$ ) should support a neoadjuvant therapy approach. Also, excluding the T1 tumors, and hence those patients with the best prognosis, may also potentially skew the overall 
A.

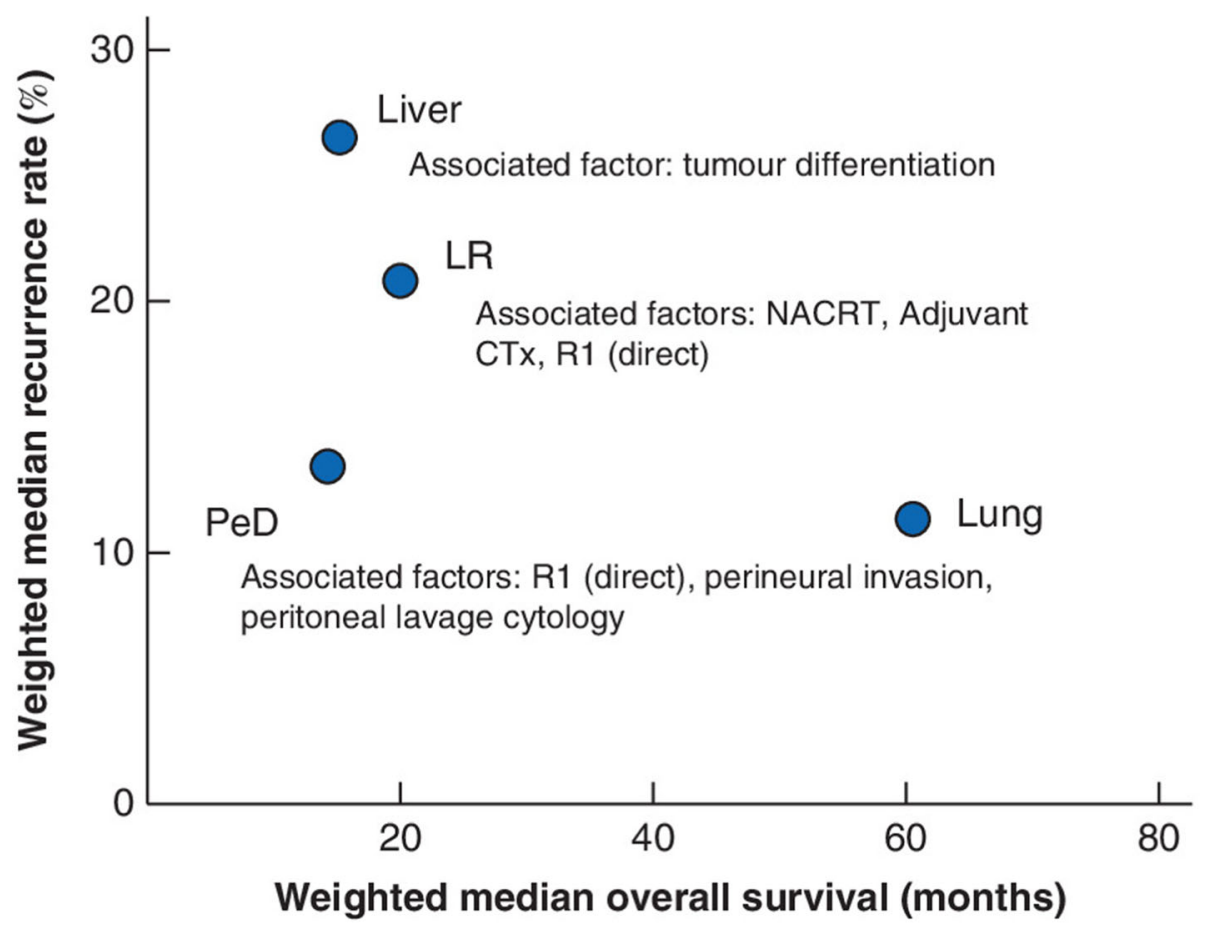

B.

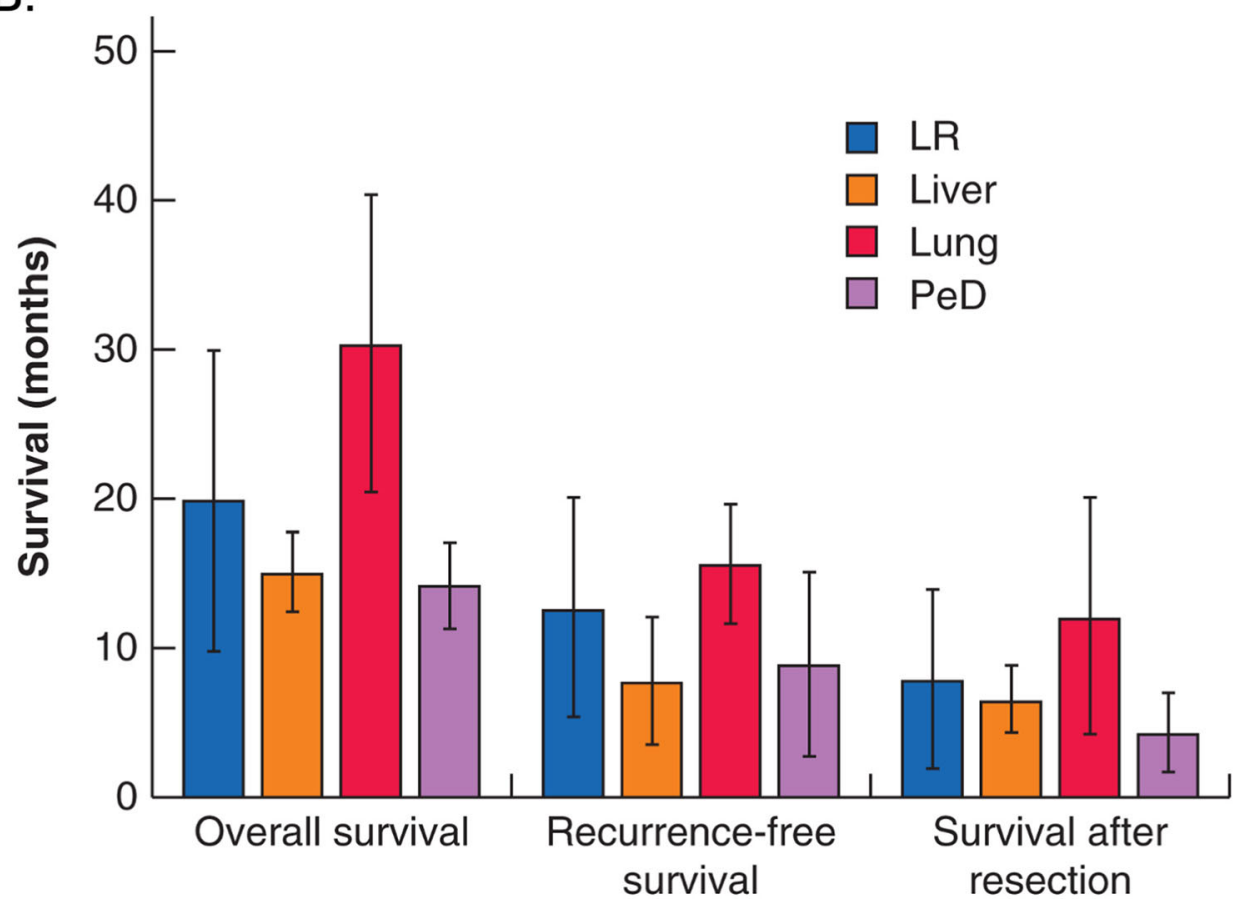


4Fig. 4 Recurrence patterns and prognosis after resection in pancreatic cancer. a Scatter diagram of recurrence patterns. b Prognosis for each site of recurrence. Outcomes were summed and weighted averages of median survival times determined. Error bars represent standard deviations. $L R$ locoregional; NACRT neoadjuvant chemoradiotherapy; $C T \mathrm{x}$ chemotherapy; $\mathrm{PeD}$ peritoneal dissemination. Reproduced with permissions from Tanaka et al. [18] Br J Surg. 2019;106(12):1590-601 Wiley (c)2019

survival in a negative direction for the PREOPANC outcomes.

Second, the trial included both upfront resectable and borderline pancreatic cancers. With a systemic disease behavior in mind, this may be appropriate. However, the criteria for "borderline resectable" in the PREOPANC trial deviates slightly from the borderline criteria suggested by others [31-33], and as recognized by the investigators in the study protocol [22]. The definition ensured uniformity among the Dutch centers but may hamper direct comparison to other studies reporting on borderline tumors based on different definitions. For example, the borderline tumors were allowed to have $>90^{\circ}$ and $\leq 270^{\circ}$ involvement of the vein, while most other definitions would restrict this to $\leq 180^{\circ}$ to define "borderline" $[31,33]$. Hence, cases that may have been defined as "locally advanced" by other definitions may have been included in the PREOPANC cohort as "borderline." On the other hand, arterial abutment was only accepted to $<90^{\circ}$ in PREOPANC, while this is also defined up to $180^{\circ}$ in other "borderline" definitions. Of note, while definitions of "borderline" may differ, there are currently no data that suggest one is better than the other. Hence, this hampers first and foremost comparison between studies (e.g., in patient selection), and better and improved biological definitions will be needed to arrive at uniform and acceptable criteria.

Lastly, the PREOPANC study used gemcitabine as drug of choice, which was based on data available at the time. Indeed, another multicenter trial from Japan has also investigated gemcitabine in the neoadjuvant setting (Prep-02/JSAP-05), albeit with added S-1 known to work in Asians but less so in other cohorts, and reported favorable outcomes for the neoadjuvant arm over upfront surgery, but currently only with data available in abstract form [34]. However, since then, FOLFIRINOX has proven to be superior both in the metastatic setting [9] and as adjuvant therapy after resection [10]. Thus, it is conceivable that more effective chemotherapy regimens will exert an improved effect on resectability and hence on disease-free and overall survival. Indeed, the PREOPANC investigators have designed the next study (PREOPANC-2 [35]) with neoadjuvant FOLFIRINOX treatment (as four cycles + four cycles in the experimental arm) against a gemcitabine + chemoradiation neoadjuvant arm (with four cycles of adjuvant gemcitabine after surgery). The inclusion of both resectable and borderline cancers (by the Dutch definition) will remain in PREOPANC-2. Also, the role of radiotherapy as part of neoadjuvant will remain open to debate.

\section{Future Directions}

The PREOPANC trial is an important step forward in the delineation of neoadjuvant therapy in pancreatic cancer, despite its equipoise in overall survival. Superiority for several of the secondary endpoints suggests a role for neoadjuvant therapy. The trial emphasizes several of the tribulations with neoadjuvant trials in pancreatic cancer [12], yet opens the opportunity for several hypothesis-generating questions and new hypotheses that may be pursued for further research [36]. The role of radiotherapy and its effect on tumor response needs to be better defined. Techniques have evolved in this field, and more precise and effective use may be anticipated but needs to be scrutinized in future trials. The influence of neoadjuvant-related toxicity from any regimen on the clinical course should be defined and compared between regimens-notably, FOLFIRINOX may be more toxic than other regimens. Any associated surgical-related morbidity such as postoperative pancreatic fistula [37] related to any given regimen should be defined as clinically important complications. Data on the patients who 


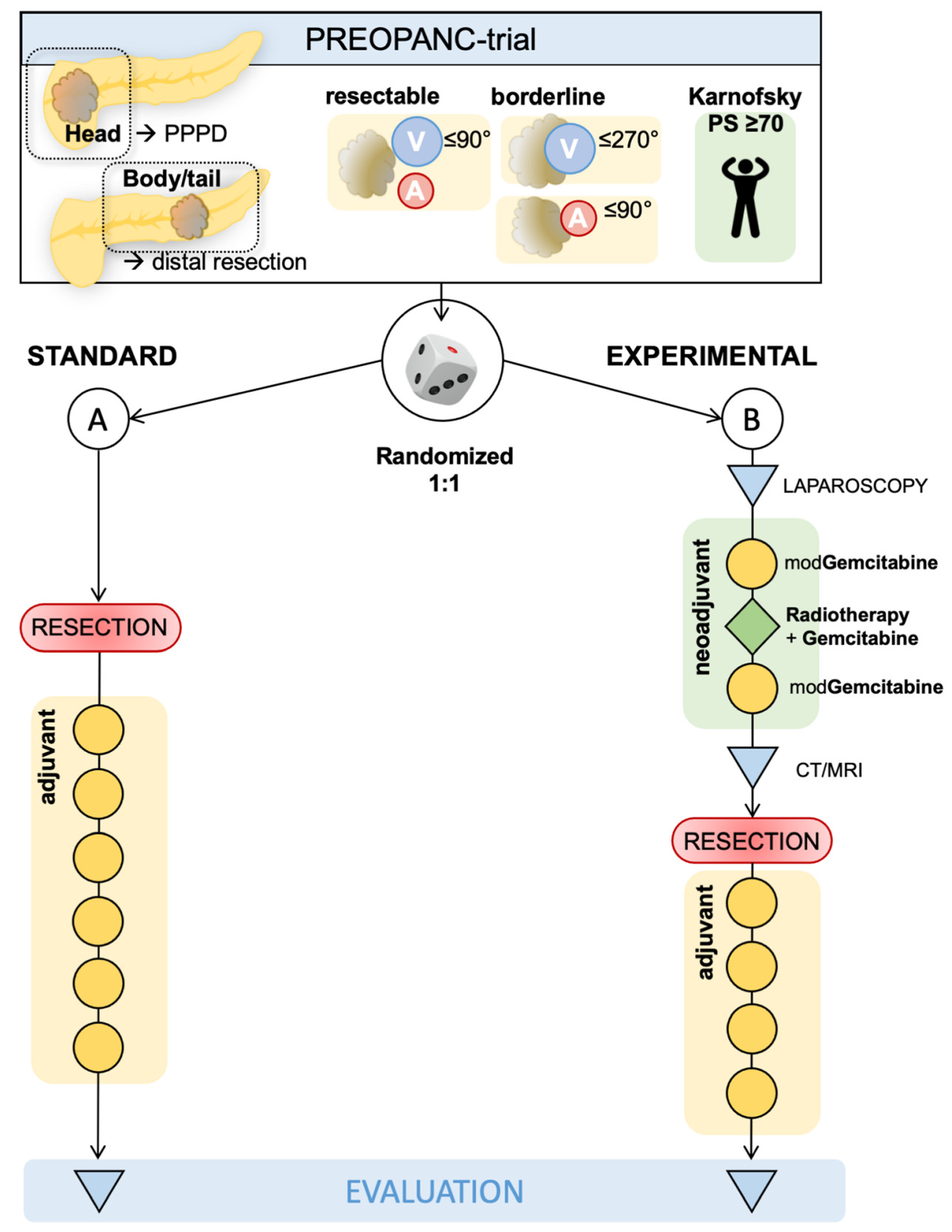

$I^{\circ}$ outcome: Overall survival by intention-to-treat (ITT) analysis

$I^{\circ}$ outcome: Diseease-free survival; local recurrence free survival; distant metastasis-free survival; resection rate; $\mathrm{R} 0$ resections; complications ++

Fig. 5 Flow scheme to illustrate the PREOPANC trial design. The full dose of gemcitabine was $1000 \mathrm{mg} / \mathrm{m}^{2} /$ dose on days 1,8 and 15 in six cycles of 28 days ( 4 weeks) each 
underwent resection in PREOPANC suggest that those receiving neoadjuvant chemoradiotherapy were at lower risk of developing post-op pancreatic fistula than those who went immediately to surgery ( 0 of $66,0 \%$ vs. 9 of $98,9.2 \%$; $\mathrm{P}=0.011$, with no differences noted for any other complications [38].

With several observational data suggesting neoadjuvant chemotherapy to be favorable $[39,40]$, the PREOPANC study [23] represents the first randomized trial to investigate this. Moving on to the PREOPANC-2 trial, the role of FOLFIRINOX against the gemcitabine-based radiochemotherapy protocol will be compared. Meta-analysis of FOLFIRINOX in borderline-resectable [41] disease suggests beneficial outcomes, and future trials on this regimen may yield a more evident outcome profile, in terms of response effect on tumors, resectability rates and survival. Furthermore, the concept of "total neoadjuvant chemotherapy," as has been addressed by others [42, 43], may be further scrutinized with this approach. Tongue in cheek, and as in the Winnie-the-Pooh quote above, maybe having the "honey and condensed milk" (total neoadjuvant) but not bothering with the bread (no adjuvant) (Fig. 2) is the suitable balance between optimal care and too much? The right timing and the best balance? And for whom? There is certainly room for future trials in this area.

While several ongoing trials will explore the exact role and give new answers as to the benefit of neoadjuvant combination regimens in the setting of operable pancreatic cancer, there is still much to be learned of the biological cancer behavior. However, with the advent of novel tumor markers, better risk stratification, delineation of molecular subgroups and personalized therapy, the future may see us design completely different trials based on "one patientone tumor-one therapy" rather than the current rough-and-tumble situation of slightly opaque definitions. With much still to be learned, there is also much to be gained from biological investigation, exploring mechanisms, molecular pathways and into the optimal therapy and the optimal strategy-towards the eventual improvement of patients with pancreatic cancer. Hence, it is only fitting to close this note off with another quote of wisdom:

"What's wrong with knowing what you know now and not knowing what you don't know until later?" from Winnie-the-Pooh by A. A. Milne

\section{ACKNOWLEDGEMENTS}

Funding. No funding or sponsorship was received for this study or publication of this article.

Authorship. All named authors meet the International Committee of Medical Journal Editors (ICMJE) criteria for authorship for this article, take responsibility for the integrity of the work as a whole, and have given their approval for this version to be published.

Disclosures. Kjetil Søreide has nothing to disclose.

Compliance with Ethics Guidelines. This article is based on previously conducted studies and does not contain any new studies with human participants or animals performed by any of the authors.

Open Access. This article is licensed under a Creative Commons Attribution-NonCommercial 4.0 International License, which permits any non-commercial use, sharing, adaptation, distribution and reproduction in any medium or format, as long as you give appropriate credit to the original author(s) and the source, provide a link to the Creative Commons licence, and indicate if changes were made. The images or other third party material in this article are included in the article's Creative Commons licence, unless indicated otherwise in a credit line to the material. If material is not included in the article's Creative Commons licence and your intended use is not permitted by statutory regulation or exceeds the permitted use, you will need to obtain permission directly from the copyright holder. To view a copy of this licence, 
visit http://creativecommons.org/licenses/bync/4.0/.

\section{REFERENCES}

1. Huang J, Lok V, Ngai CH, Zhang L, Yuan J, Lao XQ, et al. Worldwide burden of, risk factors for, and trends in pancreatic cancer. Gastroenterology. 2020. https://doi.org/10.1053/j.gastro.2020.10.007.

2. Mohammadian-Hafshejani A. The global, regional, and national burden of pancreatic cancer and its attributable risk factors in 195 countries and territories, 1990-2017: a systematic analysis for the Global Burden of Disease Study 2017. Lancet Gastroenterol Hepatol. 2019;4(12):934-47. https://doi. org/10.1016/s2468-1253(19)30347-4.

3. Søreide K, Aagnes B, Møller B, Westgaard A, Bray F. Epidemiology of pancreatic cancer in Norway: trends in incidence, basis of diagnosis and survival 1965-2007. Scand J Gastroenterol. 2010;45(1): 82-92.

00365520903358899 .

4. Klaiber U, Hackert T, Neoptolemos JP. Adjuvant treatment for pancreatic cancer. Transl Gastroenterol Hepatol. 2019;4:27. https://doi.org/10.21037/ $\operatorname{tgh} .2019 .04 .04$.

5. Neoptolemos JP, Palmer DH, Ghaneh P, Psarelli EE, Valle JW, Halloran CM, et al. Comparison of adjuvant gemcitabine and capecitabine with gemcitabine monotherapy in patients with resected pancreatic cancer (ESPAC-4): a multicentre, openlabel, randomised, phase 3 trial. Lancet. 2017;389(10073):1011-24. https://doi.org/10.1016/ s0140-6736(16)32409-6.

6. Neoptolemos JP, Moore MJ, Cox TF, Valle JW, Palmer DH, McDonald AC, et al. Effect of adjuvant chemotherapy with fluorouracil plus folinic acid or gemcitabine vs. observation on survival in patients with resected periampullary adenocarcinoma: the ESPAC-3 periampullary cancer randomized trial. JAMA. 2012;308(2):147-56. https://doi.org/10. 1001/jama.2012.7352.

7. Neoptolemos JP, Stocken DD, Bassi C, Ghaneh P, Cunningham D, Goldstein D, et al. Adjuvant chemotherapy with fluorouracil plus folinic acid vs. gemcitabine following pancreatic cancer resection: a randomized controlled trial. JAMA. 2010;304(10): 1073-81. https://doi.org/10.1001/jama.2010.1275.

8. Neoptolemos JP, Stocken DD, Tudur Smith C, Bassi C, Ghaneh P, Owen E, et al. Adjuvant 5-fluorouracil and folinic acid vs observation for pancreatic cancer: composite data from the ESPAC-1 and 3(v1) trials. Br J Cancer. 2009;100(2):246-50. https://doi.org/10.1038/sj.bjc.6604838.

9. Conroy $\mathrm{T}$, Desseigne $\mathrm{F}$, Ychou $\mathrm{M}$, Bouché $\mathrm{O}$, Guimbaud R, Bécouarn Y, et al. FOLFIRINOX versus gemcitabine for metastatic pancreatic cancer. N Engl J Med. 2011;364(19):1817-25. https://doi. org/10.1056/NEJMoa1011923.

10. Conroy T, Hammel P, Hebbar M, Ben Abdelghani $\mathrm{M}$, Wei AC, Raoul JL, et al. FOLFIRINOX or gemcitabine as adjuvant therapy for pancreatic cancer. N Engl J Med. 2018;379(25):2395-406. https://doi. org/10.1056/NEJMoa1809775.

11. Roalsø M, Aunan JR, Søreide K. Refined TNM-staging for pancreatic adenocarcinoma - real progress or much ado about nothing? Eur J Surg Oncol. 2020;46(8):1554-7. https://doi.org/10.1016/j.ejso. 2020.02.014.

12. Del Chiaro M, Søreide K. Trials and tribulations of neoadjuvant therapy in pancreatic cancer. Br J Surg. 2018;105(11):1387-9. https://doi.org/10.1002/bjs. 11003.

13. Doppenberg D, van Veldhuisen E, Busch OR, van Tienhoven G, Wilmink JW, Besselink MG. Preoperative therapy in patients with borderline resectable and locally advanced pancreatic cancer. In: Søreide K, Stättner S, editors. Textbook of pancreatic cancer - principles and practice of surgical oncology. Switzerland: Springer; 2021. p. 1514.

14. Kleive D, Vetrhus M, Silva M, Bratlie SO, Søreide K. Pancreatoduodenectomy with portal vein resection. Textbook of pancreatic cancer - principles and practice of surgical oncology. Switzerland: Springer; 2020 .

15. Belfiori G, Fiorentini G, Tamburrino D, Partelli $S$, Pagnanelli M, Gasparini G, et al. Vascular resection during pancreatectomy for pancreatic head cancer: a technical issue or a prognostic sign? Surgery. 2020. https://doi.org/10.1016/j.surg.2020.08.002.

16. Heinrich S, Besselink M, Moehler M, van Laethem JL, Ducreux M, Grimminger P, et al. Opinions and use of neoadjuvant therapy for resectable, borderline resectable, and locally advanced pancreatic cancer: international survey and case-vignette study. BMC Cancer. 2019;19(1):675. https://doi. org/10.1186/s12885-019-5889-5.

17. Versteijne E, Vogel JA, Besselink MG, Busch ORC, Wilmink JW, Daams JG, et al. Meta-analysis comparing upfront surgery with neoadjuvant treatment in patients with resectable or borderline resectable pancreatic cancer. Br J Surg. 2018;105(8): 946-58. https://doi.org/10.1002/bjs.10870. 
18. Tanaka M, Mihaljevic AL, Probst P, Heckler M, Klaiber U, Heger U, et al. Meta-analysis of recurrence pattern after resection for pancreatic cancer. Br J Surg. 2019;106(12):1590-601. https://doi.org/ 10.1002/bjs.11295.

19. Valle JW, Palmer D, Jackson R, Cox T, Neoptolemos JP, Ghaneh P, et al. Optimal duration and timing of adjuvant chemotherapy after definitive surgery for ductal adenocarcinoma of the pancreas: ongoing lessons from the ESPAC-3 study. J Clin Oncol. 2014;32(6):504-12. https://doi.org/10.1200/jco. 2013.50.7657.

20. Mayo SC, Gilson MM, Herman JM, Cameron JL, Nathan H, Edil BH, et al. Management of patients with pancreatic adenocarcinoma: national trends in patient selection, operative management, and use of adjuvant therapy. J Am Coll Surg. 2012;214(1):33-45. https://doi.org/10.1016/j. jamcollsurg.2011.09.022.

21. Fisher AV, Ma Y, Wang X, Campbell-Flohr SA, Rathouz PJ, Ronnekleiv-Kelly SM, et al. National trends in centralization of surgical care and multimodality therapy for pancreatic adenocarcinoma. J Gastrointest Surg. 2020;24(9):2021-9. https://doi. org/10.1007/s11605-019-04361-3.

22. Versteijne E, van Eijck $\mathrm{CH}$, Punt CJ, Suker M, Zwinderman AH, Dohmen MA, et al. Preoperative radiochemotherapy versus immediate surgery for resectable and borderline resectable pancreatic cancer (PREOPANC trial): study protocol for a multicentre randomized controlled trial. Trials. 2016;17(1):127. https://doi.org/10.1186/s13063016-1262-z.

23. Versteijne E, Suker M, Groothuis K, AkkermansVogelaar JM, Besselink MG, Bonsing BA, et al. Preoperative chemoradiotherapy versus immediate surgery for resectable and borderline resectable pancreatic cancer: results of the dutch randomized phase III PREOPANC Trial. J Clin Oncol. 2020;38(16):1763-73. https://doi.org/10. 1200/jco.19.02274.

24. Delayre T, Guilbaud T, Resseguier N, Mamessier E, Rubis M, Moutardier V, et al. Prognostic impact of tumour-infiltrating lymphocytes and cancer-associated fibroblasts in patients with pancreatic adenocarcinoma of the body and tail undergoing resection. Br J Surg. 2020;107(6):720-33. https:// doi.org/10.1002/bjs.11434.

25. Dreyer SB, Jamieson NB, Upstill-Goddard R, Bailey PJ, McKay CJ, Biankin AV, et al. Defining the molecular pathology of pancreatic body and tail adenocarcinoma. Br J Surg. 2018;105(2):e183-91. https://doi.org/10.1002/bjs.10772.
26. Birnbaum DJ, Bertucci F, Finetti P, Birnbaum D, Mamessier E. Head and body/tail pancreatic carcinomas are not the same tumors. Cancers (Basel). 2019;11(4):4-6. cancers11040497.

27. Tomasello G, Ghidini M, Costanzo A, Ghidini A, Russo A, Barni S, et al. Outcome of head compared to body and tail pancreatic cancer: a systematic review and meta-analysis of 93 studies. J Gastrointest Oncol. 2019;10(2):259-69. https://doi.org/10. 21037/jgo.2018.12.08.

28. van Erning FN, Mackay TM, van der Geest LGM, Groot Koerkamp B, van Laarhoven HWM, Bonsing $\mathrm{BA}$, et al. Association of the location of pancreatic ductal adenocarcinoma (head, body, tail) with tumor stage, treatment, and survival: a populationbased analysis. Acta Oncol. 2018;57(12):1655-62. https://doi.org/10.1080/0284186x.2018.1518593.

29. Muralidhar V, Nipp RD, Mamon HJ, Punglia RS, Hong TS, Ferrone C, et al. Association between very small tumor size and decreased overall survival in node-positive pancreatic cancer. Ann Surg Oncol. 2018;25(13):4027-34. https://doi.org/10.1245/ s10434-018-6832-8.

30. Ansari D, Bauden M, Bergström S, Rylance R, Marko-Varga G, Andersson R. Relationship between tumour size and outcome in pancreatic ductal adenocarcinoma. Br J Surg. 2017;104(5):600-7. https://doi.org/10.1002/bjs.10471.

31. Kang MJ, Jang JY, Kwon W, Kim SW. Clinical significance of defining borderline resectable pancreatic cancer. Pancreatology. 2018;18(2):139-45. https://doi.org/10.1016/j.pan.2017.12.003.

32. Zaky AM, Wolfgang CL, Weiss MJ, Javed AA, Fishman EK, Zaheer A. Tumor-vessel relationships in pancreatic ductal adenocarcinoma at multidetector CT: different classification systems and their influence on treatment planning. Radiographics. 2017;37(1):93-112. https://doi.org/10.1148/rg. 2017160054

33. Denbo JW, Fleming JB. Definition and management of borderline resectable pancreatic cancer. Surg Clin North Am. 2016;96(6):1337-50. https://doi.org/10. 1016/j.suc.2016.07.008.

34. Unno M, Motoi F, Matsuyama Y, Satoi S, Matsumoto I, Aosasa S, et al. Randomized phase II/III trial of neoadjuvant chemotherapy with gemcitabine and S-1 versus upfront surgery for resectable pancreatic cancer (Prep-02/JSAP-05). J Clin Oncol. 2019;37(4):189. https://doi.org/10. 1200/JCO.2019.37.4_suppl.189.

35. Janssen QP, Homs MYV, Wilmink JW, Van Tienhoven G, Besselink MG, Koerkamp BG. 
Neoadjuvant FOLFIRINOX versus neoadjuvant chemoradiotherapy and adjuvant chemotherapy for (borderline) resectable pancreatic cancer: update on the PREOPANC-2 study. HPB. 2020;22:S277. https://doi.org/10.1016/j.hpb.2020.04.191.

36. Søreide K, Sund M. Translational research in surgical oncology. Br J Surg. 2017;104(5):491-2. https:// doi.org/10.1002/bjs.10467.

37. Søreide K, Healey AJ, Mole DJ, Parks RW. Pre-, periand post-operative factors for the development of pancreatic fistula after pancreatic surgery. HPB (Oxford). 2019;21(12):1621-31. https://doi.org/10. 1016/j.hpb.2019.06.004.

38. van Dongen JC, Suker M, Versteijne E, Bonsing BA, Mieog JSD, de Vos-Geelen J, et al. Surgical complications in a multicenter randomized trial comparing preoperative chemoradiotherapy and immediate surgery in patients with resectable and borderline resectable pancreatic cancer (PREOPANC Trial). Ann Surg. 2020. https://doi.org/10.1097/sla. 0000000000004313.

39. Gamboa AC, Rupji M, Switchenko JM, Lee RM, Turgeon MK, Meyer BI, et al. Optimal timing and treatment strategy for pancreatic cancer. J Surg Oncol. 2020;122(3):457-68. https://doi.org/10. 1002/jso.25976.
40. Watson MD, Thompson KJ, Musselwhite LW, Hwang JJ, Baker EH, Martinie JB, et al. The treatment sequence may matter in patients undergoing pancreatoduodenectomy for early stage pancreatic cancer in the era of modern chemotherapy. Am J Surg. 2020. https://doi.org/10.1016/j.amjsurg.2020. 10.030 .

41. Janssen QP, Buettner S, Suker M, Beumer BR, Addeo $\mathrm{P}$, Bachellier $\mathrm{P}$, et al. Neoadjuvant FOLFIRINOX in patients with borderline resectable pancreatic cancer: a systematic review and patient-level metaanalysis. J Natl Cancer Inst. 2019;111(8):782-94. https://doi.org/10.1093/jnci/djz073.

42. Kim RY, Christians KK, Aldakkak M, Clarke CN, George B, Kamgar M, et al. Total neoadjuvant therapy for operable pancreatic cancer. Ann Surg Oncol. 2020. https://doi.org/10.1245/s10434-02009149-3.

43. Murphy JE, Wo JY, Ryan DP, Jiang W, Yeap BY, Drapek LC, et al. Total neoadjuvant therapy with FOLFIRINOX followed by individualized chemoradiotherapy for borderline resectable pancreatic adenocarcinoma: a phase 2 clinical trial. JAMA Oncol. 2018;4(7):963-9. https://doi.org/10.1001/ jamaoncol.2018.0329. 